\title{
Myocardial ischemia with normal coronary angiography in a chronic kidney disease patient
}

\author{
Chrissa Sioka ${ }^{1}$, Konstantinos Papadimitropoulos ${ }^{1}$, Lampros Michalis ${ }^{2}$, \\ Konstantinos Pappas ${ }^{2}$, Lampros Lakkas ${ }^{2}$, Andreas Fotopoulos ${ }^{1}$, Evangelia Dounousi ${ }^{3}$ \\ ${ }^{1}$ Department of Nuclear Medicine, Medical School, University Hospital of Ioannina, Greece \\ ${ }^{2}$ Department of Cardiology, Medical School, University Hospital of Ioannina, Greece \\ ${ }^{3}$ Department of Nephrology, Medical School, University Hospital of Ioannina, Greece
}

A 66-year-old male patient with end-stage chronic kidney disease (CKD), underwent renal transplant in 1997 that subsequently failed due to chronic allograft nephropathy, necessitating his return to hemodialysis. Since 2013 the patient was followed-up with myocardial perfusion imaging (MPI SPECT) because of atypical periodic chest discomfort but negative electrocardiogram and clinical examination. While the patient, being asymptomatic in successive MPI SPECT performed in the years 2013, 2014, 2016, and 2017 demonstrated progressive ischemia of the inferior/ inferolateral cardiac wall and part of the myocardial apex, only partially reversible at rest. Bulls-eye displayed the perfusion abnormalities and assessed the affected myocardium. In the upper bulls-eye images (Fig. 1) a normal MPI SPECT is depicted in another individual for comparison, and in the lower images, the MPI SPECT of the present case demonstrated perfusion defects. The coronary arteries that perfuse each myocardial territory were also indicated. Coronary angiogram in 2017 showed no hemodynamically significant coronary abnormalities (Fig. 1), however myocardial blush was very low, indicating small vessels dysfunction. Due to a very small probability of significant epicardial stenosis no intravascular ultrasound or optical coherence tomography were performed.

Similar results were obtained by echocardiography which demonstrated a left ventricle with marginal systolic function, concentric hypertrophy and progressive hypokinesia of the mid-inferior septal and mid-inferior lateral regions and akinesia of basal inferior segments (Suppl. Video S1, 2012, 2016, 2017). Moreover, as part of the initial exam in 2013, a dipyridamole stress echocardiogram was performed in order to assess coronary flow reserve, which was relatively small in this patient (2.4).

The etiology of the discordant results in the present patient between MPI SPECT and angiography may be due to an irregular vasoconstrictor/ vasodilatory coronary capability. Thus, episodes of increased coronary vasoconstriction may have resulted in progressive development of myocardial ischemia without obvious permanent coronary stenoses.

Conflict of interest: None declared

Address for correspondence: Chrissa Sioka, MD, PhD, Department of Nuclear Medicine, University Hospital of Ioannina, 1 Stavrou Niarchou Street, Ioannina 45110, Greece, tel: +30-26510-07220, e-mail: csioka@yahoo.com 


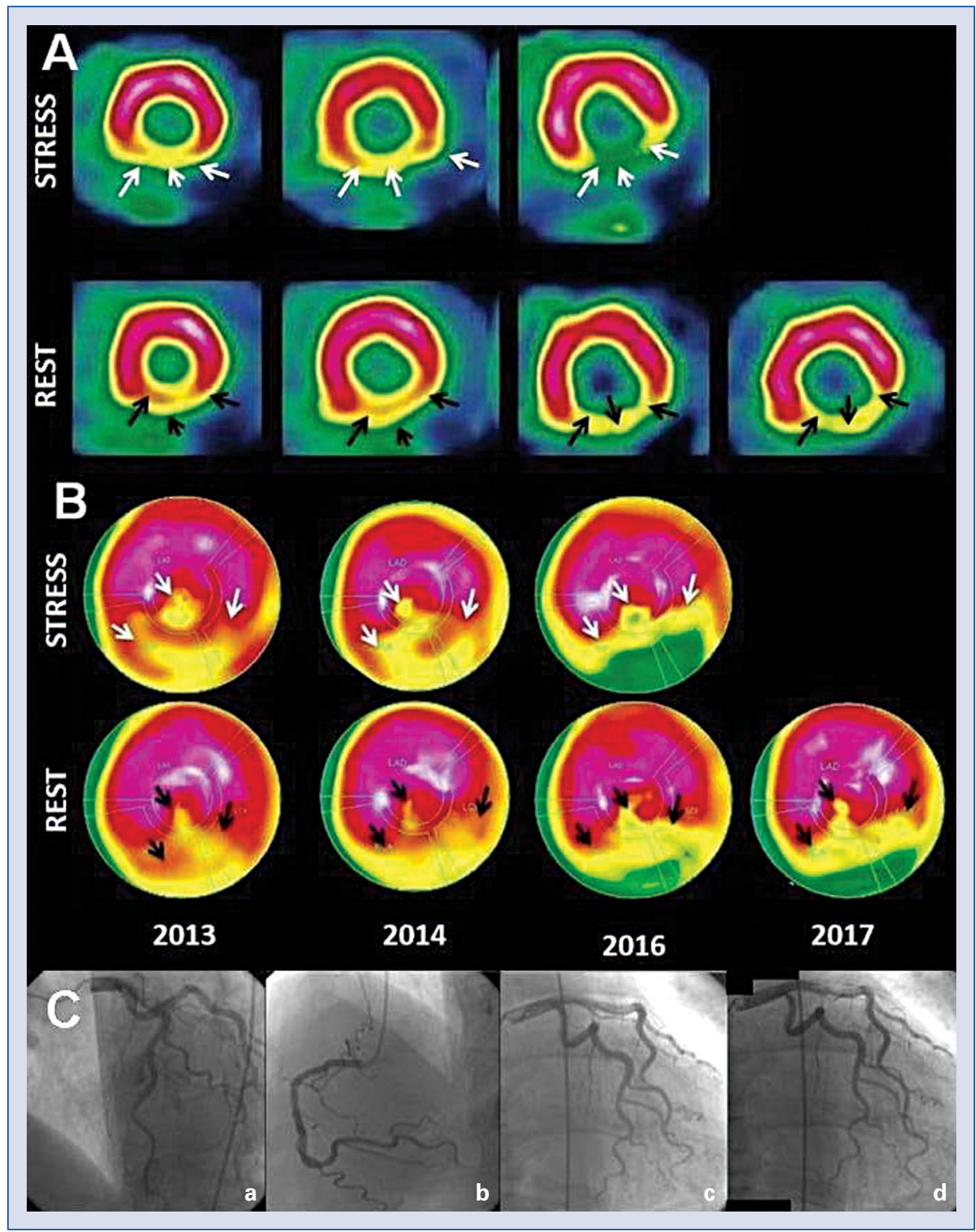

Figure 1. A. Shows the myocardium in the short axis; B. Bulls-eye myocardium perfusion images (MPI), depicting the entire myocardium. MPI scans were performed with a 1 day protocol using Technetium $\left({ }^{99} \mathrm{mTc}\right)$ Tetrofosmin in years, $2013,2014,2016$, and 2017. Black arrows point to the hypoperfused areas during stress and the white arrows indicate at rest. The differences between the stress and at rest MPI demonstrate the progressive nature of the myocardial ischemia which appears to be less severe and reversible at rest in 2013 and more severe and partially reversible in 2016 and 2017. The parts of the ischemic myocardium predominantly involve the inferior, inferolateral and a segment of the apical wall (seen in the center of the Bulls-eye image); $\mathbf{C}$. Coronary angiography (a: left coronary artery; $\mathbf{b}$ : right coronary artery; c: circumflex artery; d: circumflex artery with myocardial blush). There is no stenosis in any of the coronary arteries and with low myocardial blush. 
